\author{
Military Technical College \\ Kobry El-Kobbah, \\ Cairo, Egypt.
}

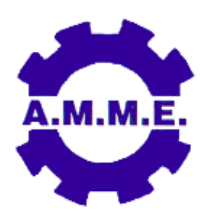

$17^{\text {th }}$ International Conference on Applied Mechanics and Mechanical Engineering.

\title{
COMPUTER AIDED TRADITION JIGS AND FIXTURES DESIGN
}

\author{
H.M.A Hussein ${ }^{1}$, A. Mahrous ${ }^{2}$, A. F. Barakat ${ }^{3}$ and O.M. Dawood ${ }^{4}$
}

\begin{abstract}
Jigs and fixtures is a branch of science including many of mechanical design and production engineering rules. The traditional concepts of jigs and fixtures have famous applications until now in the Egyptian industries. There are many CAD applications found in the worldwide literature, but all of them related to modular fixtures and other applications of flexible fixtures. Most of the modular fixture CAD systems ignore the relation between clamp force and the machining cutting force. In this paper, a new system framework discusses a CAD in traditional jigs and fixture. The proposed system package includes visual basic and AutoCAD. It extracts and recognizes the machined part features (cylindrical or prismatic shapes) and starts the jigs design automatically. The system classifies the machined parts into groups and for each group suggests the optimum jigs design.
\end{abstract}

\section{KEY WORDS}

Fixture Design, Process Planning, Feature Recognition, STEP AP 203. 


\section{INTRODUCTION}

Jigs and fixtures are machine tools aided devices which deal with machine such as turning, milling, drilling, grinding, and broaching machines. Welding and assembly fixtures are other branches of fixtures which has no deals with machine tools. Jigs are machine shop devices that include means of tool guiding; they are mostly applicable to operations performed on drilling machine, e.g. drilling or reaming. Fixtures are special holding devices that aided to hold the workpiece firmly and easy, they may include means of setting the cutter. Fixtures are used for milling, turning, grinding and similar manufacturing operation.

The traditional structure design of jigs and fixtures include 3 main items, guides, locators, and clamps. The guide bush's used to guide the tool to the accurate position of machining operation. The locators used to constrain the workpiece in its accurate position inside the fixture. The locator constrains the workpiece in about 3 to 5 degree of freedom. Finally, the clamp is used to constrain the last 6th degree of freedom of the workpiece. The clamp force must be designed to withstand the cutting operation. In the traditional fixture design technique, the workpiece is first study together with the part process plan, the surface roughness and the machining remarks for the previous machining operation must be one of the important knowledge for the jigs and fixtures designer. The individual's knowledge based design items accumulate together to form the optimum jigs and fixture design [1].

The traditional technique of jigs and fixture design have a good relation with the machine element design, as the device mainly depend on the standard parts such as bolts, dowel pins, cams, ..etc.

Modular and flexible fixtures are another branch of jigs and fixtures which are completely standard parts. These kinds of devices are detachable, it means that all of its components can be assembly and dis-assembly, and there is no component will be throughout or retired. The jig plate is a horizontal or vertical drilled plate, and the standard locators and clamps are plugged in those drilled holes. The modular fixtures are mainly used with CNC machines. There is no need to calculate the clamp force in this case. Also, the position of plugging the locators and clamps keep as a problem during assembly the modular fixture. This technique has the same results of the traditional fixture, but simultaneously, it is completely deferent from it $[2,3]$.

Computer Aided fixture design is software which mainly used with the modular fixture. There are about hundreds of research works made to cover some problems during the selection or assemble of the modular fixture using computer facilities. There is no research work found in the literature to cover the traditional jigs and fixture technique [4,5].Traditional jigs and fixture design need many calculation modules to cover many items in the fixture design, such as; feature recognition, clamping force, cam design, locator position, guide position, selection of fixture standard components, design and modeling of the complete jigs and fixture, process planning, ...etc.

In the proposed system, the part (prismatic or cylindrical) are first drawn on any CAD system and saved as STEP files extension. The data are extracted from the selected file using tailor made software. A feature recognition rules are pre-prepared to check 
the resulted extracted data and decide which data shape will represent a machined feature. A process planning module is prepared to define the good process steps of the machined part. The previous machined surface or the tolerances surface cannot automatically determined by the system until now, and they are selected manually. The system includes many databases for the standard components of the jigs and fixtures, so, the optimum standard components are selected to suite the target part and to design the other nonstandard jigs components automatically such as jig plate and walls. The system is constructed using visual basic program which connected with EWDraw as add-on module. The system is connected with AutoCAD which represents the graphical media in the proposed package.

The main target of the proposed system is to construct a used friendly system for automated design of traditional jigs and fixtures.

\section{GEOMETRIC DATA EXCHANGE}

In design and manufacturing, many systems are used to manage technical product data. Each system has its own data formats so; the same information has to be entered multiple times into multiple systems leading to redundancy and errors. The problem is not unique to manufacturing but more acute because design data are complex and 3D leading to increase the scope for errors and misunderstanding between operators. From the literature, many solutions have been proposed. The most successful ones have been standards for data exchange. The data exchange formats include Standard Exchange Transfer (SET) in France; Verband Der Automobilindustrie-Flachen-Schnittstelle (VDAFS) in Germany, Drawing Transfer File (DXF) and Initial Graphics Exchange Specification (IGES) in the USA. These standards have success in transferring the data between various CAD systems but they have limitations in transferring the product data from CAD to CAM applications [6].

The limitation is due to the ability of current CAD systems to transfer all the information required for CAM applications. To overcome these difficulties, the International Standards Organization (ISO) produces an International Standard to almost include all aspects of technical product data and named STEP for the Standard for Product Model Data. Nearly every major CAD/CAM system now contains a module to read and write data defined by one of the STEP Application Protocols (APs). In the USA, the most commonly implemented protocol is called AP203. This protocol is used to exchange data describing designs represented as solid models and assemblies of solid models. In Europe, a very similar protocol called AP214 performs the same function [7].

\section{MAIN PROCESSES OF THE PROPOSED SYSTEM}

The proposed system framework is build using visual Basic and connected to AutoCAD. A commercial tailor-made build-in module called EWdrew is installed on Visual Basic. The visual basic receives the part shape in STEP format and shows it on the EWDrew standalone screen. Visual Basic extracts the part data and features from the machined part step drawing using a build in rules. Also, visual Basic builds 
the part process planning for the part. A group technology for many parts shape stored in the program database in shape of script file, which one of the AutoCAD customization tools. AutoCAD works in this package as graphical media to present the final jigs and fixtures final design resulted from the proposed system.

In the proposed system, extracting the part feature data from STEP AP-203 is according to ISO 10303-203:2011. The system works to cover 2 main shapes of the machined parts, the prismatic parts [8] and the cylindrical parts [9]. In both of the prismatic or the cylindrical parts shapes, the system is passes through the same sequence as usually used in the literature, these sequence are 1. Data extraction, 2. Feature recognition, 3. Process Planning, 4. Fixture Design.

\section{Data Extraction Technique}

The object-oriented approach is used for extracting the part data from STEP AP 203 in another neutral file format using Visual basic programming language. The significance of using STEP AP 203 file is its compatibility with several known CAD systems. Among different geometric representation techniques, Boundary Representation (B-Rep) is used. The data extraction file will be generated by relevant data extraction from STEP AP203 file. The procedure is illustrated in Fig. 1 [10]. The significance of the technique is to represent the part as B-rep structure along with its geometric properties necessary to perform downstream manufacturing applications like feature recognition, process planning and fixture planning.

\section{Feature Extraction}

A Feature in computer aided design CAD software can be called a region of a part with some interesting geometric or topological patterns. This meaning can refer to all sorts of information, such as, shape, functional or manufacturing information. Although many types of features have been investigated, the most common type of feature is the form feature, which contains both shape information and parametric information. Examples of form features common in many shape models are round holes, steps, slots, bosses, and pockets,....etc, in case or prismatic parts, and groove, undercut, chamfer round corners, shoulder, taper, ... etc, in case of cylindrical parts $[10,11]$.

Features are generic shapes with which engineers associate certain attributes and knowledge useful in reasoning about the product. Features encapsulate the engineering significance of portions of the geometry which, are important in product design, product definition, and reasoning for a variety of applications.

Features are majorly classified as prismatic and rotational features [10,11]. Prismatic features are further categorized into depression and boss features. The depression features are further subdivided into two categories i.e., flat and cylindrical. The flat features are step, slot and pocket features that can be through, blind and round corners. 


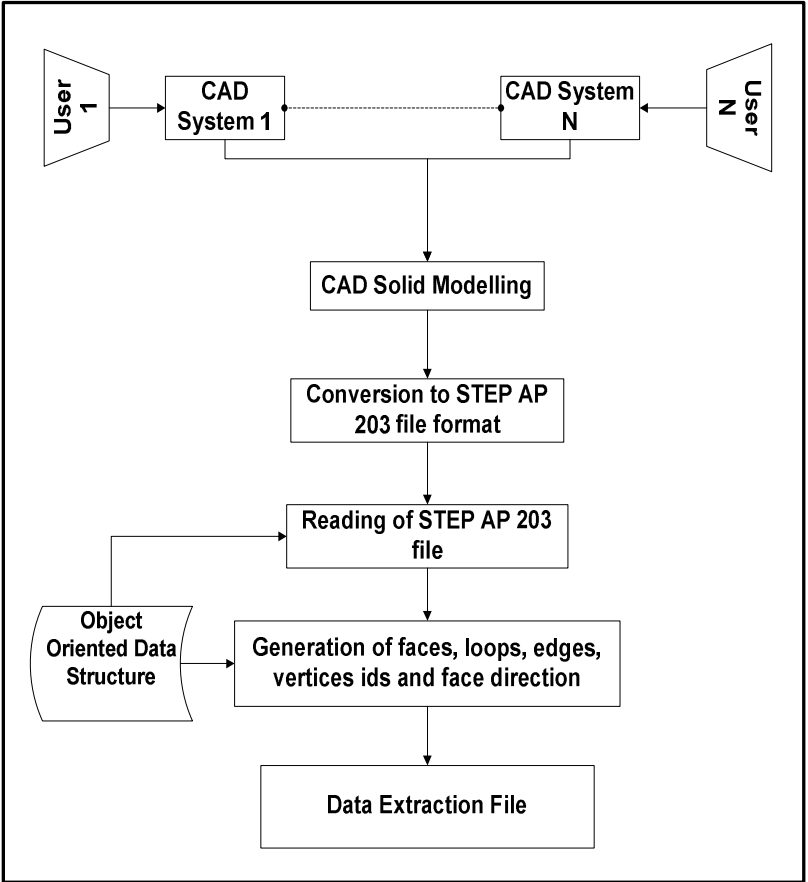

Fig. 1. Geometric Data Extraction [10].

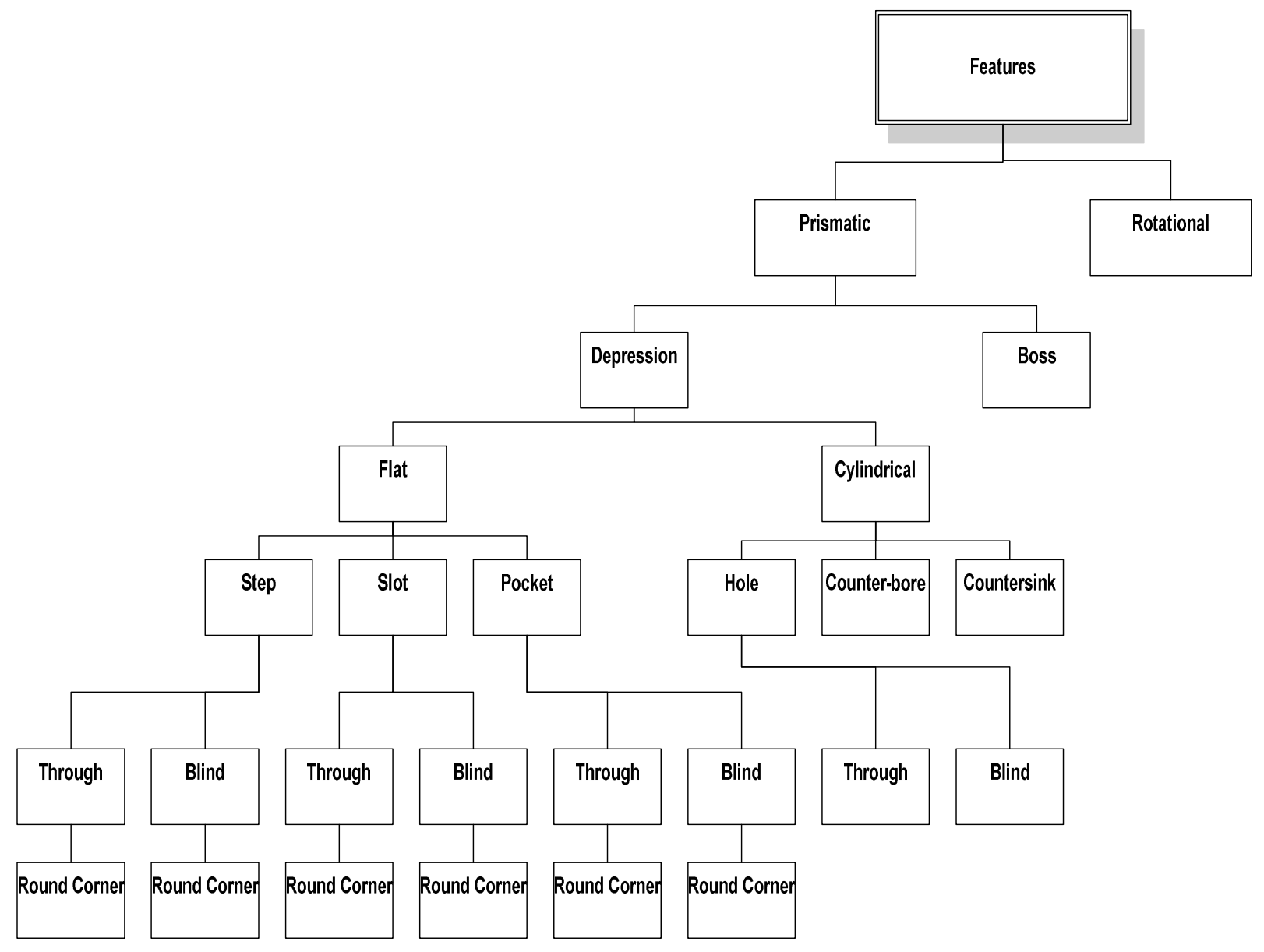

Fig 2. Classification of Prismatic Features [10]. 
The cylindrical features which related to the flat or prismatic features are hole, counter-bore and countersink features. The classification can be shown in Fig. 2 . The cylindrical or rotational features which belong to the rotational machined parts are shown in Fig. 3 [11].

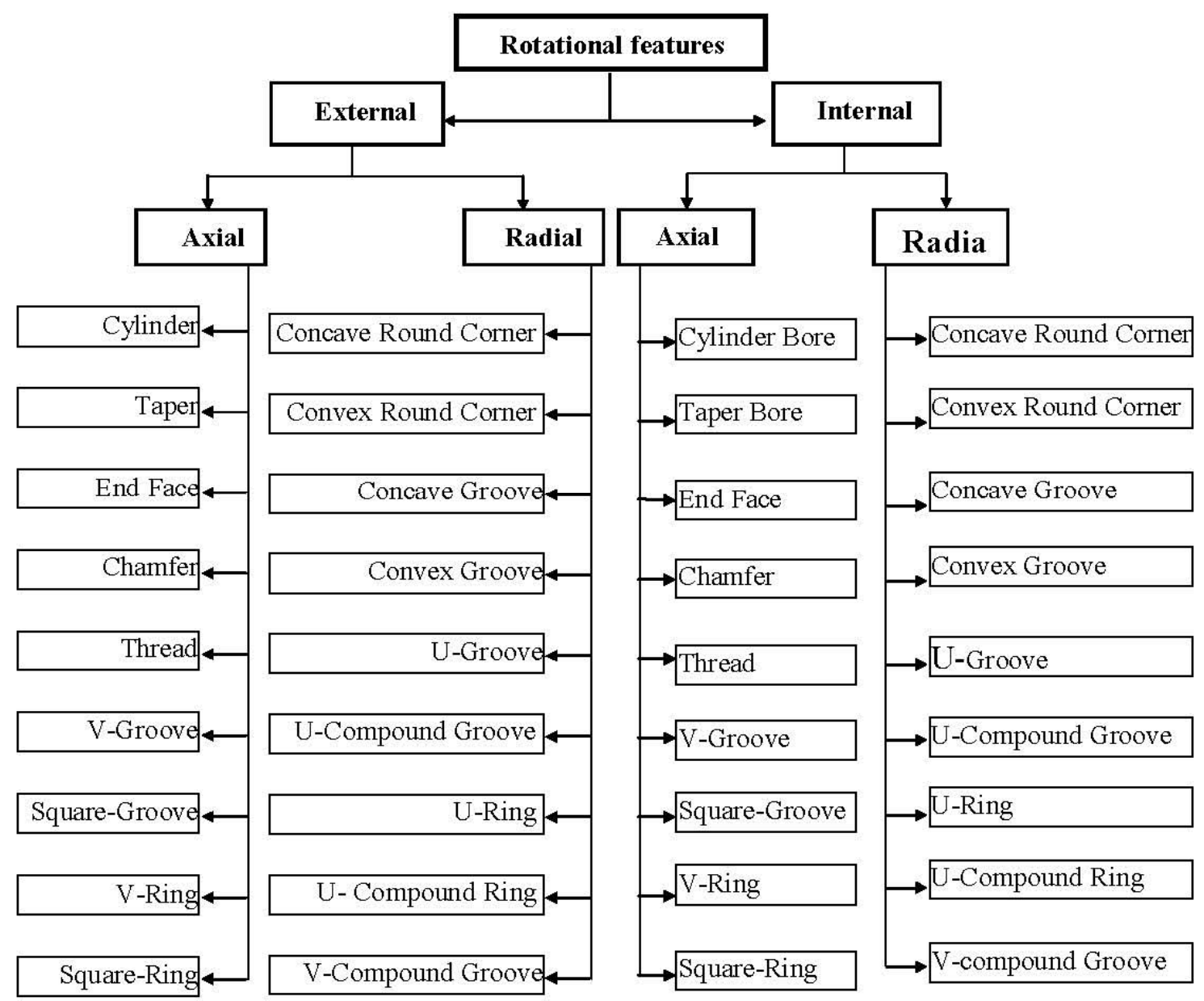

Fig 3. Classification of Cylindrical / Rotational Features [11].

\section{Process Planning}

For each of the 2 machined parts, the program recognizes the part shape (prismatic or rotational part) from the STEP file database, and then, the program starts to construct the suitable process plan for the part based on its features and the flowchart designed by the program designer.

\section{Fixture Design}

Fixture module is depending on the process planning module. In Which, The system checks the machined part and automatically recognizes the features required for fixture design. The program has 2 options, automatic, and manual. In case of automatic option, the program opens the AutoCAD graphical media and starts the draw of each suggested fixture on a separate group of AutoCAD layers which belong 
to the fixture code. This automated operation performed depending on the sequence received from the process planning module. In case of manual operation, the program let the user to select the required process needed for fixture design. Once the user selects the process, the program sequence run on the part in its defined stage and draws the fixture for this process only. The main program interface for the proposed program is shown in Fig.4a with a cylindrical part and in Fig.4b for prismatic part.

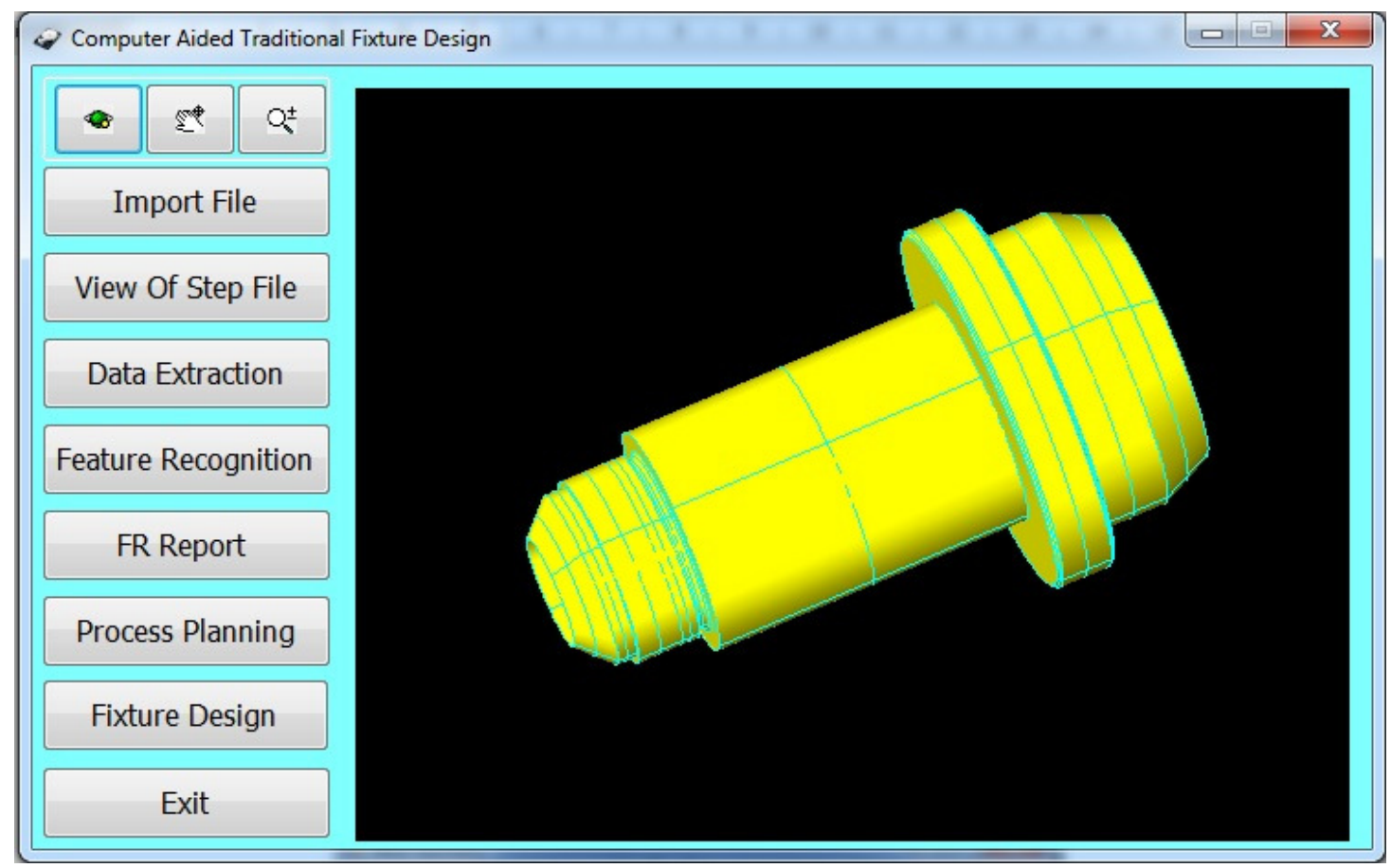

Fig 4a. The program interface with application on cylindrical part.

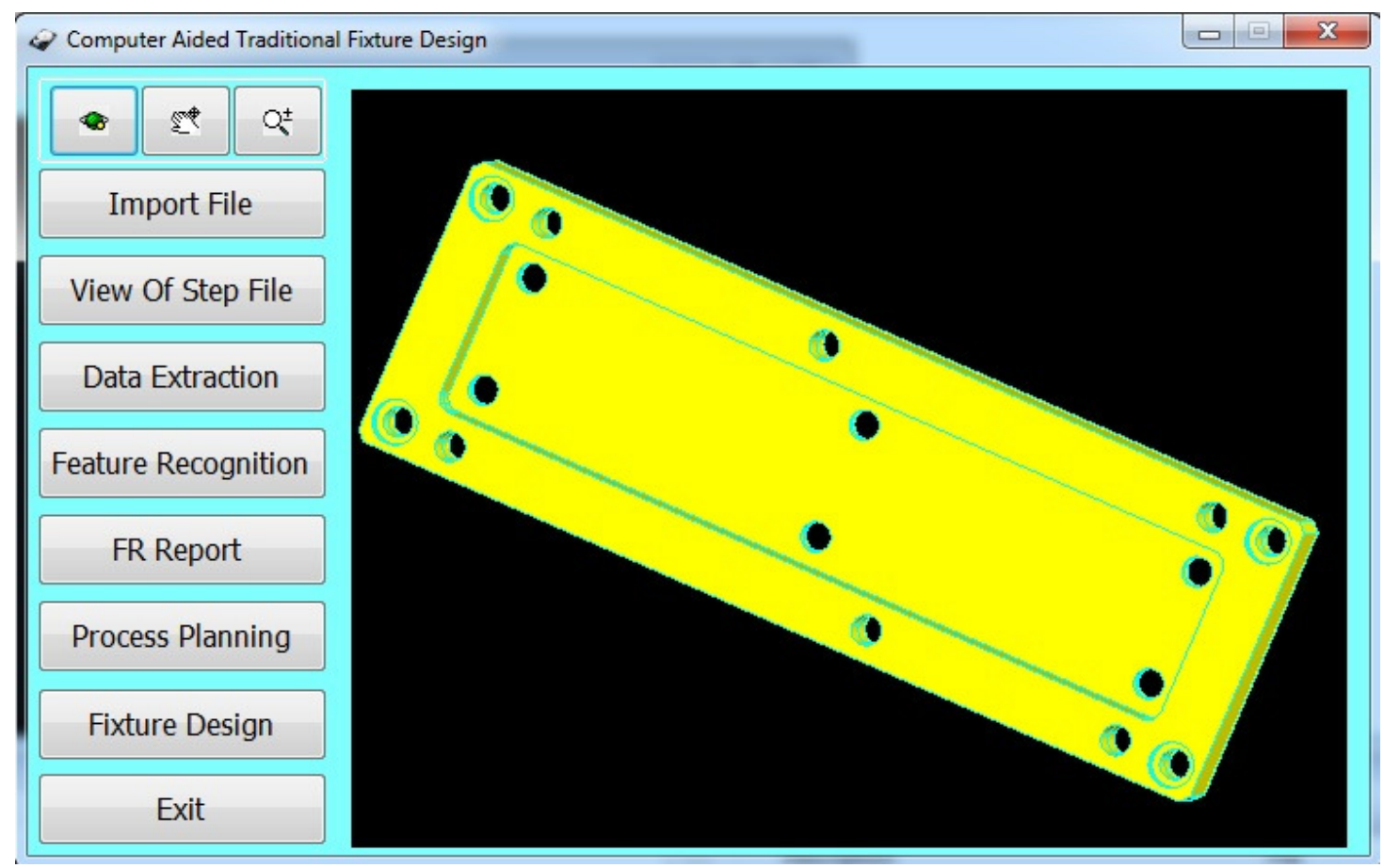

Fig 4b. the program interface with application on Prismatic part. 


\section{CASE STUDY}

The part shown in Fig. 5 represents the manual drawing and dimensions of a cylindrical part with flange. The required operation is to drill (in the flange) the 4 holes of $12 \mathrm{~mm}$ Dia. The fixture design will do manually for this operation. First, the program runs and extracts the data from the STEP file; the program library includes a group of part families, and the corresponding optimum jigs and fixture designs for these parts. For each, the program searches for the necessary dimensions and reference surface to install the optimum fixture design parametrically. If the extracted part data from the STEP file meets the part design code, which stored inside the program, then the program starts searching about those dimensions and surface roughness. Second, the program defines the machined feature rules for the cylindrical part to create the optimum process planning which required for machine the part. The program dialogue box for the case study part is shown in Fig. 6 . When, the user selects the required operation for drilling the 4 holes, the program checks the previous machined operation which is the inner boring for the Dia 60. For this surface, the program checks for the suitable locator dimension from the locators database.

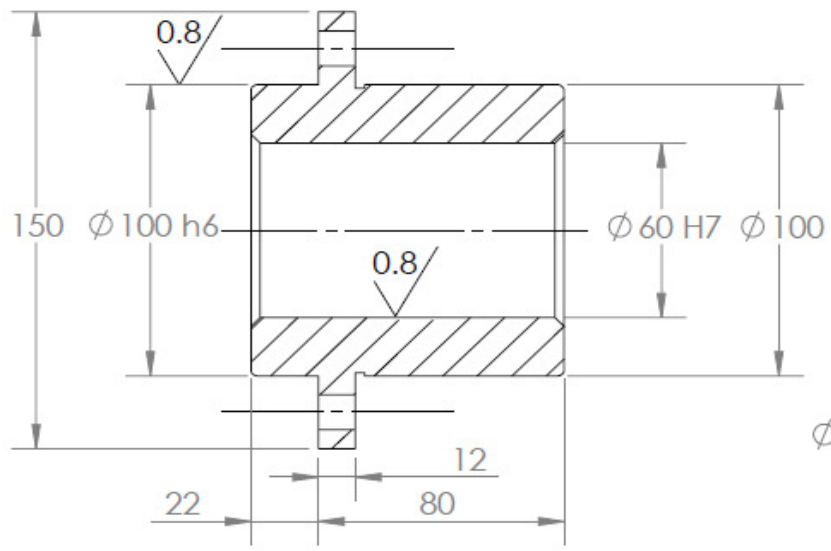

SECTION B-B

SCALE $1: 2.5$

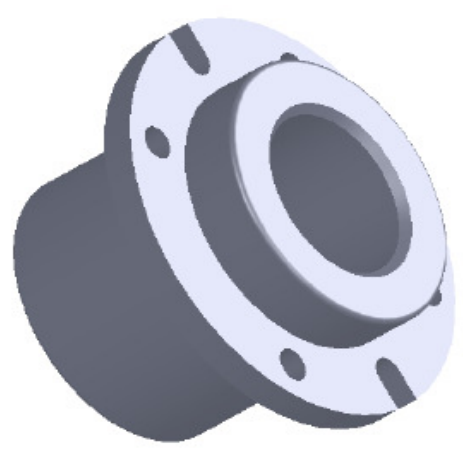

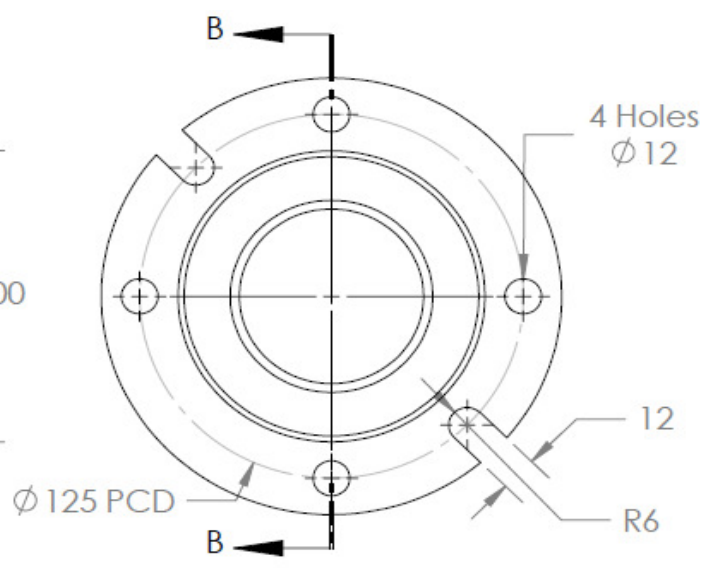

3.2/ $0.8 /$

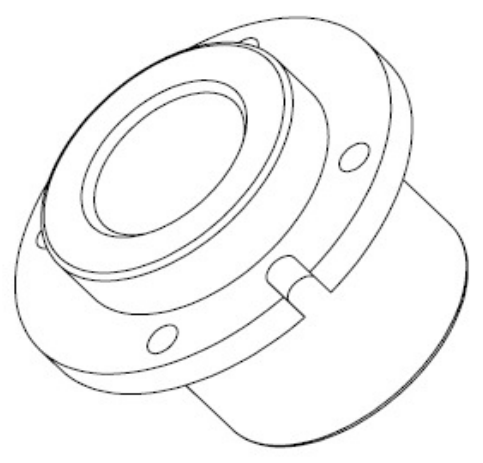

Fig. 5. Drawing and dimensions of the case study part.

Next, the program checks the holes, and selects the optimum guide bush from the guide bush database. The optimum distance between the jig plate and upper position of the part is a rule, which recorded in the program database for this part code. The 
program checks the upper surface of the part to be a reference for determining the level of the jig plate. The position of the guide bushes depend on the level of the jig plate. Next, the program selects the optimum clamp device, which selects from the clamp graphical database. The clamp force is determined based on the cutting operation force and moment. Finally, the whole design of the optimum jigs or fixtures design is parametrically done under AutoCAD graphical media. The program group technology database designed to extend and accept any number of part designs in the future.

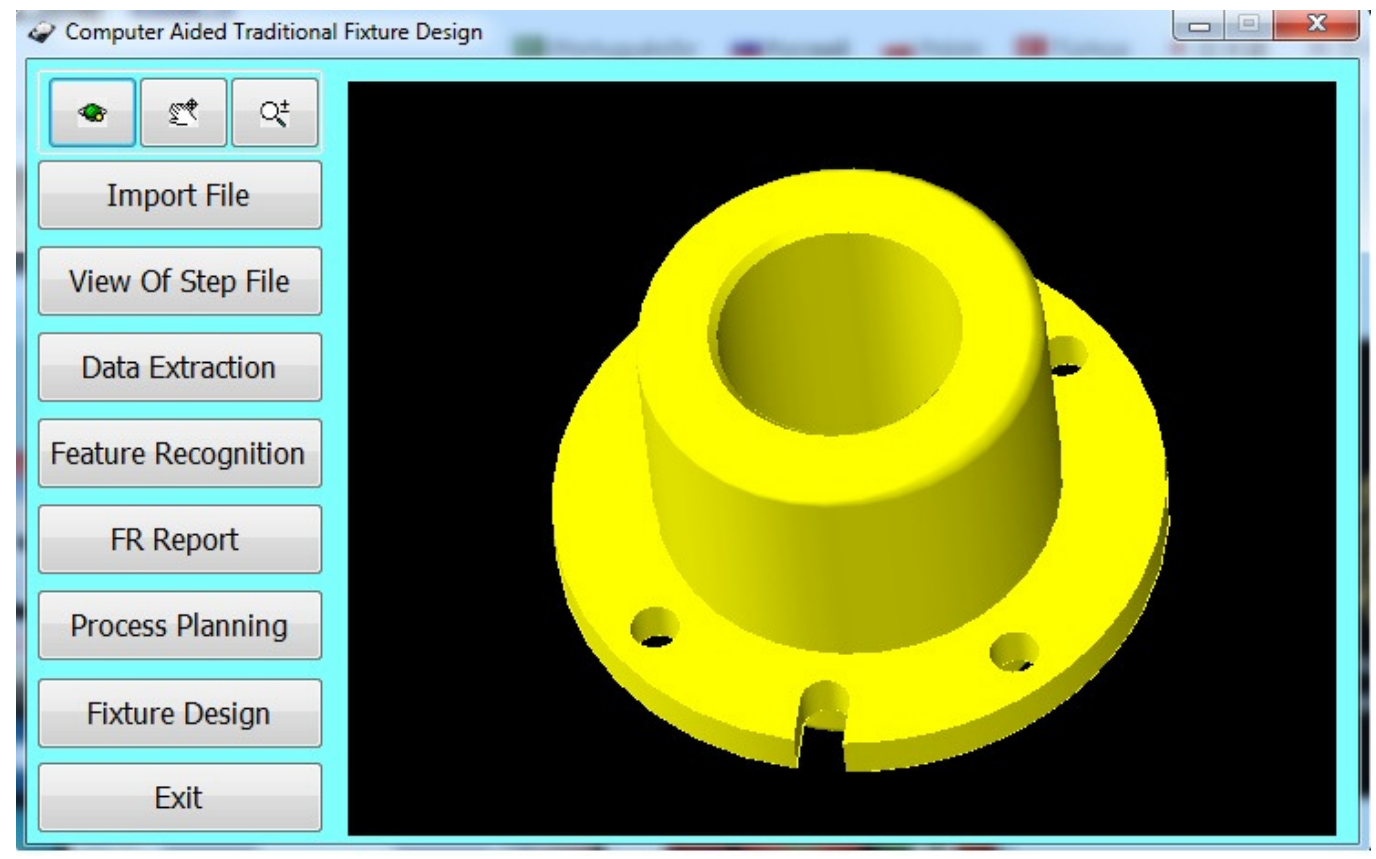

Fig 6. The program dialogue box for the case study part.

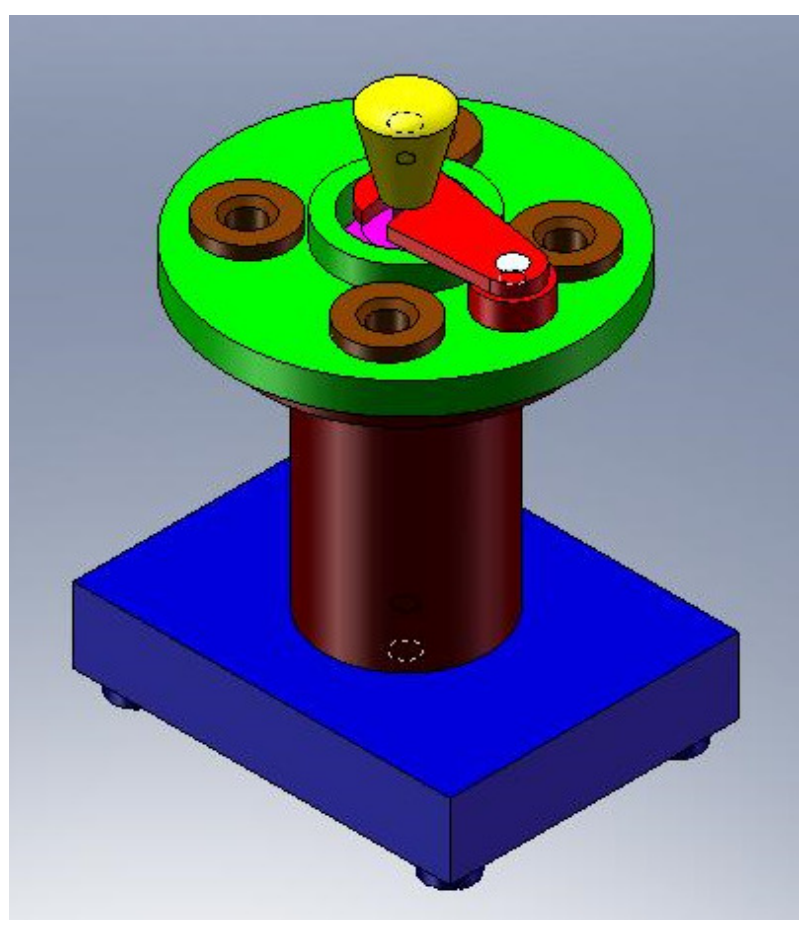

Fig 7. The output resulted fixture design. 
The AutoCAD script file (one of the AutoCAD customization tools) controls the drafting operations of the fixture design. First, it create a number of layers which suit the fixture design components, for example; layer for locator, layer for guide bushes, and layer for clamps. Layers for each machined components in the fixture design. The program insert the standard parts in its position into the AutoCAD graphical media related the position of the machined part. Next, the AutoCAD starts draft automatically, the other non-standard fixture components.

Finally, the complete fixture design is drafted parametrically and automatically on the AutoCAD graphical media. Figure 7 shows the resulted fixture design for the case study part.

\section{CONCLUSIONS}

The design of traditional jigs and fixtures still has a critical demand in the Egyptian factories. The proposed system framework is a very useful tool to handle this industrial demand. The proposed framework design concept has the ability to handle many machined parts (prismatic and cylindrical shapes) from the industry. For this reason, it can be useful in factories which have specified products, and the other factories which have verities of products. The program is designed based on the visual basic program, and the EWDrew module add-on to the visual basic. This module includes a standalone graphical media which read the part database in its extension even the iges [12] or the stp. This add-on application gives a powerful to visual basic in the industrial applications.

\section{REFERENCES}

[1] P.H. Joshi, "Jigs and fixtures - Design Manual", Second Edition, ISBN-0-07140556-9, McGrawHill, 2003.

[2] A.Y.C. Nee, K. Whybrew, and A. Senthil kumar "Advanced Fixture Design for FMS", Advanced Manufacturing Series, ISBN-13:978-1-4471-2119-0, SpringerVerlag London Limited, 1995.

[3] Y. Rong, Y. Zhu, "Computer Aided Fixture Design", ISBN-0-8247-9961-5, Marcel Dekker, Inc., 1999.

[4] A.Y.C. Nee, Z.J. Tao, A. Senthil Kumar, "An Advanced Treatise on Fixture Design and Planning", Series On Manufacturing Systems And Technology, ISBN 981-256-059-9, World Scientific, 2004.

[5] Y. Rong, S. H. Huang, Z. Hou, "Advanced Computer-aided Fixture Design", ISBN: 0-12-594751-8, Elsevier Academic Press, 2005.

[6] E.S. Abouel Nasr, A.K. Kamrani, "Computer-Based Design and Manufacturing An Information-Based Approach", ISBN 9780387233239, Springer Verlag, 2007.

[7] Xun Xu - A.Y.C. Nee, "Advanced Design and Manufacturing Based on STEP", Springer Series in Advanced Manufacturing, ISBN 978-1-84882-738-7, Springer-Verlag London Limited, 2009. 
[8] E.S. Abouel Nasr, A.A. Khan, A. Alahmari , H.M.A. Hussein, "A Feature Recognition System Using Geometric Reasoning", International Conference on Manufacture of Lightweight Components, ManuLight2014, Dortmund, Germany, 3-4th April 2014, Also Published in :in Procedia CIRP vol. 18, pp.238-243, 2014.

[9] H.M.A. Hussein, E.S. Abouel Nasr and A.A. Khan, "Automated Feature Extraction from Cylindrical Parts Based on STEP, Sustainable Intelligent Manufacturing International Conference, SIM2013, Leiria, Lisbon, Portugal, Green design, Materials and Manufacturing Processes, Taylor \& Francis (ISBN: 978-1-138-00046-9), pp.233-238, 26-29 June, 2013.

[10] A.A. Khan, "An Integrated System for Automatic Computer Aided Fixture Planning and Fixture Design", PhD Thesis, Industrial Engineering Department, Faculty of engineering, King Saud University, 2014.

[11] A.B. Abdulghafour, M.A. Al-Bassam, "Automatic Features Recognition for Symmetrical Shapes.", Eng. \& Tech. Journal, Vol. 30, No. 12 , 2012.

[12] E.S. Abouel Nasr, H.M.A. Hussein, A.E. Ragab A.K. Kamrani, " A FeatureBased Approach To An Integrated CAD/CAPP System In Sheet Metal Blanking Dies.", International Journal of Rapid Prototyping, Vol. 4, Nos. 2/3/4, 2014. 